\title{
HERMITIAN FORMS AND HIGHER ALGEBRAIC $K$-THEORY
}

\author{
BY CHARLES H. GIFFEN ${ }^{1}$
}

\author{
Communicated by Hyman Bass, May 18, 1977
}

Introduction. This note promulgates certain invariants for Hermitian forms over a ring with antistructure which are defined utilizing the algebraic $K$-theory of the ring in a $Z_{2}$-equivariant fashion. Low degree cases of these invariants extend the classical rank, discriminant, and Hasse-Witt invariants.

The principal result described here is that these invariants (using all degrees) yield the stable classification of forms over many fields and algebras for which low degree cases alone do not usually suffice (because of signature invariants that may be defined). The manner in which this is accomplished is quite amusing, in that it sheds light on why the reductions of the signatures modulo certain powers of 2 are in fact determined by the low degree invariants. More general results can be obtained, e.g. for higher unitary algebraic $K$-theory. However, these are suppressed here in favor of an outline of the equivariant algebraic $K$ theory upon which this work so crucially relies. This will be dealt with at length in $\left[\mathbf{G i}_{\mathbf{2}}\right]$, and arithmetic aspects will be pursued further elsewhere.

1. Hermitian forms. If $R$ is a ring and $M_{R}$ is the category of right $R$-modules, then $P_{R}$ will denote the full, exact subcategory of $M_{R}$ determined by the finitely generated projective modules, and $K_{n} R, n \in \mathbf{Z}$, will denote the (GerstenWagoner) algebraic $K$-theory groups of $R$ (cf. [Ge]).

Recall that an antistructure $(\alpha, u)$ on $R$ consists of an antiautomorphism $r \mapsto r^{\alpha}$ of $R$ and a unit $u \in R^{\times}$such that $u u^{\alpha}=1$ and $r^{2}=u u^{-1}, r \in R$. An Hermitian form over $(R, \alpha, u)$, or an $(\alpha, u)$-form over $R$, is a pair $(\mathbf{P}, \lambda)$ with $\mathbf{P} \in P_{R}$ and $\lambda: \mathbf{P} \times \mathbf{P} \rightarrow R$ such that

$$
\lambda(x, y+z r)=\lambda(x, y)+\lambda(x, z) r, \lambda(y, x)=\lambda(x, y)^{\alpha} u \quad \text { for } x, y, z \in \mathbf{P}, r \in R,
$$

and $(\operatorname{Ad} \lambda): \mathbf{P} \rightarrow D_{\alpha} \mathbf{P}$ is an isomorphism where $(\operatorname{Ad} \lambda)(x)(y)=\lambda(x, y), x, y \in$ $R$. Here, $D_{\alpha}: P_{R} \rightarrow P_{R}$ is the $\alpha$-conjugate duality cofunctor $D_{\alpha}$ ? $=\operatorname{Hom}_{R}($ ?, $R)$ where $\operatorname{Hom}_{R}(\mathbf{P}, R)$ has right $R$-module structure $(f \circ r)(x)=r^{\alpha} f(x), x \in \mathbf{P}, r \in$ $R, f \in D_{\alpha} \mathbf{P}$. There are evident notions of isometry and orthogonal direct sum of $(\alpha, u)$-forms over $R$ (cf. [Wa]), resulting in a permutative category $U(R, \alpha, u)$ and (Gersten-Wagoner type) unitary algebraic $K$-theory groups $K U_{n}(R, \alpha, u), n$ $\in \mathbf{Z}$, of $(R, \alpha, u)$. Of primary interest here is $K U_{0}(R, \alpha, u)$ which is just the Grothendieck group of $U(R, \alpha, u)$.

AMS (MOS) subject classifications (1970). Primary 10C05, 18F25; Secondary 55B20.

${ }^{1}$ Research supported by the NSF, the Institute for Advanced Study, and a University of Virginia Sesquicentennial Research Associateship. 
2. Equivariant algebraic $K$-theory. Refined and adapted from [Va], the following is essential to this study.

THEOREM A. If $R$ is a ring with antistructure $(\alpha, u)$, then there is a $Z_{2}$. spectrum $\mathbf{K}_{\alpha, u} R$, functorial in $(R, \alpha, u)$, defining a $Z_{2}$-equivariant generalized cohomology theory $K_{\alpha, u} R_{Z_{2}}$ ( ) such that:

(1) as ordinary spectrum $\mathrm{K}_{\alpha, u} R$ is an $\Omega$-spectrum for $K_{*} R$, e.g. $K_{\alpha, u} R_{Z_{2}}^{-n}\left(Z_{2}\right)=K_{n} R, n \in \mathrm{Z}$, where $Z_{2}$ has free $Z_{2}$-action;

(2) the fixed point subspectrum $\left(\mathbf{K}_{\alpha, u} R\right)^{Z_{2}}$ is an $\Omega$-spectrum for $K U_{*}(R, \alpha, u), e . g . K_{\alpha, u} R_{Z_{2}}^{-n}(\mathrm{pt})=K U_{n}(R, \alpha, u), n \in \mathrm{Z}$;

(3) the map pt $\leftarrow Z_{2}$ of $Z_{2}$-spaces induces the forgetful natural transformation $\varphi_{*}: K U_{*}(R, \alpha, u) \rightarrow K_{*} R$.

Note. Equivariant cohomology theories are as in $[\mathrm{Br}]$.

3. Invariants of forms. Let $D^{p-1} K_{n}(R, \alpha, u)=K_{\alpha, u} R_{Z_{2}}^{n}\left(S_{A}^{p-1}\right)$ where $S_{A}^{p-1}$ denotes the $(p-1)$-sphere with antipodal involution, and set $\hat{D K}_{n}=\lim _{p}$ $D^{p-1} K_{n}$. If $\widehat{D K}_{n}$ and $K U_{n}$ are filtered by

$$
F^{p} \widehat{D K}_{n}=\operatorname{Ker}\left(\widehat{D K}_{n} \rightarrow D^{p-1} K_{n}\right) \text { and } F^{p} K U_{n}=\operatorname{Ker}\left(K U_{n} \rightarrow D^{p-1} K_{n}\right) \text {, }
$$

the latter induced by pt $\leftarrow S_{A}^{p-1}$, then pt $\leftarrow S_{A}^{\infty-1}$ induces imbeddings

$$
\varphi_{n}^{(p)}: K U_{n} / F^{p+1} K U_{n} \hookrightarrow \widehat{D K}_{n} / F^{p+1} \widehat{D K}_{n} \subseteq D^{p} K_{n}
$$

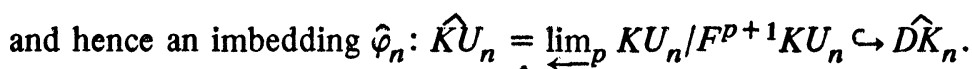

If $G^{p}=F^{p} / F^{p+1}$, then $G^{p} D K_{n}=E_{\infty}^{p,-n-p}$ where $E_{r}^{p,-q}$ is a BredonAtiyah-Hirzebruch spectral sequence for $\widehat{D K} K_{*}$ with

$$
E_{2}^{p,-q}(R, \alpha, u)=H^{p}\left(Z_{2} ; K_{q} R\right)
$$

and $K_{q} R$ has $Z_{2}$-action coming from $D_{\alpha}$. Hence, restriction $\bar{\varphi}_{n}^{(p)}$ of $\varphi_{n}^{(p)}$ imbeds $G^{p} K U_{n}(R, \alpha, u)$ in the subquotient $E_{\infty}^{p,-n-p}$ of $H^{p}\left(Z_{2} ; K_{n+p} R\right)$.

TheOREM B. For $p=0,1,2, \bar{\varphi}_{0}^{(p)}$ is equivalent to the rank, discriminant, total Hasse-Witt invariant, respectively. (Compare $\left[\mathbf{G i}_{1}\right]$.)

THEOREM C. If $F^{\infty}=\bigcap_{p} F^{p}$, then $F^{\infty} K U_{0}(E, \alpha, u)=0$ provided $E$ is (1) an archimedean or local field (locally compact) and $\alpha$ is continuous, (2) a finite or global field, or (3) a finite dimensional semisimple algebra over one of these fields ( $\alpha$ continuous if archimedean or local).

THEOREM D. If $E$ is a finite dimensional simple Q-algebra with antistructure $(\alpha, u)$, if $v$ is a real place of centre $(E)$ such that $\left(E_{v}, \alpha_{v}, u\right)$ is of type $O(\mathbf{R})$, $U(\mathbf{C})$, or $\operatorname{Sp}(\mathbf{H})$ with associated signature $\sigma_{v}: K U_{0}(E, \alpha, u) \rightarrow Z$, and if $\mathbf{F}=\mathbf{R}$, $\mathbf{C}$, or $\mathbf{H}$ respectively, then (1) there exist

$$
\tau_{v}^{(p)}: D^{p} K_{n}(E, \alpha, u) \rightarrow K_{\mathrm{F}}^{n}\left(\mathbf{R}^{p}\right)
$$


with $\hat{\tau}_{v}=\lim \tau_{v}^{(p)}$ defined such that, if $(\mathbf{P}, \lambda) \in U(E, \alpha, u)$ with $\operatorname{rank}_{v}=\rho$ and $\sigma_{v}=\sigma$, then

$$
\hat{\tau}_{v} \hat{\varphi}_{0}[\mathrm{P}, \lambda]=\frac{\rho+\sigma}{2}+\frac{\rho-\sigma}{2}\left[\gamma_{\mathrm{F}}^{1}\right] \in K_{\mathrm{F}}^{0}\left(\mathbf{R} P^{\infty}\right)=\mathrm{Z} \oplus \hat{\mathbf{Z}}_{2}
$$

where $\gamma_{\mathbf{F}}^{1}=\gamma_{\mathbf{R}}^{1} \otimes_{\mathbf{R}} \mathbf{F}, \gamma_{\mathbf{R}}^{1}$ the canonical line bundle, and

$$
\operatorname{Coker}\left(\sigma_{v} \mid F^{p+1} K U_{0}(E, \alpha, u)\right)=Z / 2^{1+\beta(F, p)},
$$

where

$$
\beta(\mathbf{F}, p)= \begin{cases}{\left[\frac{p+7}{8}\right]+\left[\frac{p+6}{8}\right]+\left[\frac{p+1}{4}\right]} & \text { if } \mathbf{F}=\mathbf{R}, \\ {\left[\frac{p+1}{4}\right]+\left[\frac{p+3}{8}\right]+\left[\frac{p+2}{8}\right]} & \text { if } \mathbf{F}=\mathbf{C}=\mathbf{H} .\end{cases}
$$

Corresponding results hold over Dedekind domains, e.g.

$$
K U_{0}(\mathrm{Z}, 1,1) / F^{p+1} K U_{0}\left(\mathrm{Z}_{\mathrm{z}} 1,1\right)=\mathrm{Z} \oplus \mathrm{Z} / 2^{\beta(\mathrm{R}, p)}, \quad p \geqslant 0 .
$$

Ideally, $F^{\infty} K U_{n}=0$ and $\hat{\varphi}_{n}: \hat{K}_{n} \cong \widehat{D K}_{n}$ would always hold, and for "arithmetic" rings these appear to be, respectively, true and plausible. For locally compact fields the "continuous algebraic $K$-theory" analogues work out nicely.

\section{BIBLIOGRAPHY}

[Br] G. E. Bredon, Equivariant cohomology theories, Lecture Notes in Math. no 34 Springer-Verlag, Berlin and New York, 1967.

[Ge] S. M. Gersten, On the spectrum of algebraic K-theory, Bull. Amer. Math. Soc. 78 (1972), 216-220.

[Gi $\mathrm{G}_{1}$ C. $\mathrm{H}$. Giffen, Hasse-Witt invariants for $(\alpha, u)$-reflexive forms and automorphisms. I, J. Algebra 44 (1977), 434-456.

$\left[\mathrm{Gi}_{2}\right]-$ Unitary and equivariant generalised algebraic $\mathrm{K}$-cohomology theory, Proceedings Conf. on homotopy theory, Northwestern University, Evanston, Ill., 1977 (to appear).

[Va] R. N. Vance, Homotopy equivariant group actions and Atiyah-real algebraic $\mathbf{K}$ theory, Ph.D. Thesis, University of Virginia, 1974.

[Wa] C. T. C. Wall, On the axiomatic foundations of the theory of Hermitian forms, Proc. Cambridge Philos. Soc. 67 (1970), 243-250.

SCHOOL OF MATHEMATICS, INSTITUTE FOR ADVANCED STUDY, PRINCETON, NEW JERSEY 08540

DEPARTMENT OF MATHEMATICS, UNIVERSITY OF VIRGINIA, CHARLOTTESVILLE, VIRGINIA 22901 